\title{
The Behavior of Normality when Iteratively Finding the Normal to a Line in an $l_{p}$ Geometry
}

\author{
Joshua M. Fitzhugh, David L. Farnsworth \\ School of Mathematical Sciences, Rochester Institute of Technology, Rochester, USA \\ Email: JMF7126@rit.edu, DLFSMA@rit.edu
}

Received October 20, 2013; revised November 20, 2013; accepted November 27, 2013

Copyright (C) 2013 Joshua M. Fitzhugh, David L. Farnsworth. This is an open access article distributed under the Creative Commons Attribution License, which permits unrestricted use, distribution, and reproduction in any medium, provided the original work is properly cited.

\begin{abstract}
The normal direction to the normal direction to a line in Minkowski geometries generally does not give the original line. We show that in $l_{p}$ geometries with $p>1$ repeatedly finding the normal line through the origin gives sequences of lines that monotonically approach specific lines of symmetry of the unit circle. Which lines of symmetry that are approached depends upon the value of $p$ and the slope of the initial line.
\end{abstract}

Keywords: Minkowski Geometry; Geometric Construction; Iteration; Normality; $l_{p}$ Geometry; Radon Curve

\section{Introduction}

Minkowski geometries are completely characterized by their unit circle, which is centrally symmetric about the origin and convex [1: p. 17]. The spaces are homogeneous (all points are the same) and generally anisotropic (the yard stick for distance is not the same in all directions). Our principal interest is the planar Minkowski $l_{p}$ geometries with $p \geq 1$. Their unit circles are

$$
|x|^{p}+|y|^{p}=1 \text {. }
$$

The exponent $p$ must be at least 1 in order for the unit circle to be convex. Convexity is required for the triangle inequality [1: pp. 22,23]. If $p=2$, this is Euclidean geometry. If $p=1$, the circle is not strictly convex. As discussed in Section 2, since in Minkowski geometries a necessary and sufficient condition for uniqueness of normal directions to lines is that the unit circle be strictly convex, we do not consider the $p=1$ case. Convex unit circles are strictly convex if they contain no line segments. The $l_{1}$ geometry is well studied and is sometimes called taxicab, Manhattan, or city-block geometry [2]. Since the unit circle for the limiting case $p \rightarrow \infty$ is the square with vertices $( \pm 1, \pm 1)$, we do not consider that geometry, as well. Figure 1 shows some $l_{p}$ unit circles and the circle with $p=0.5$, which does not produce a Minkowski geometry since the circle is not convex.

The unit circle determines distances. For the Minkowski distance between points $P_{1}$ and $P_{2}$, consider line
$L$ through the origin $O$ and parallel to the line through $P_{1}$ and $P_{2}$. The distance between $P_{1}$ and $P_{2}$ is the quotient of

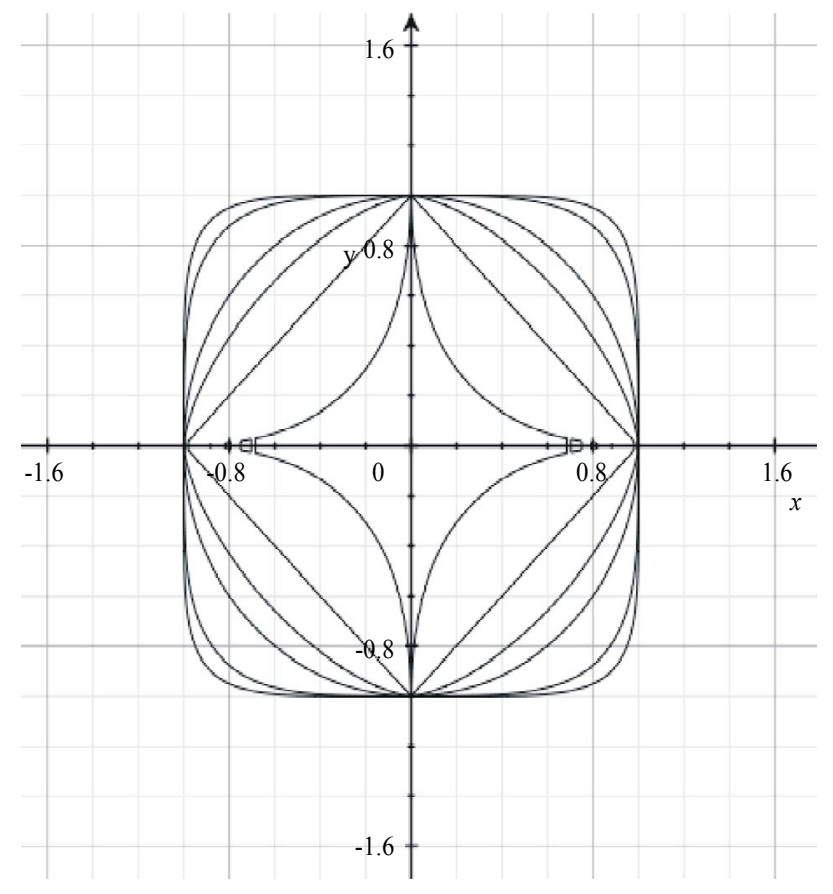

Figure 1. Unit circles $|x|^{p}+|y|^{p}=1$ for $p=0.5,1,1.5,2,4$ and 6. The circles with $p=1,1.5,2,4$ and 6 give Minkowski geometries. 
the Euclidean distance between $P_{1}$ and $P_{2}$ and the unit of measurement or scale in the direction of $L$. The unit of measurement is the Euclidean distance from the $O$ to point $Q$ where $L$ intersects the unit circle [3: p. 225, 4]. Equivalently, translate the axes so that the origin is at $P_{1}$ and the point $P_{2}$ has coordinates $\left(x_{2}, y_{2}\right)$. The Minkowski distance between points $P_{1}$ and $P_{2}$ is the value of $d>0$ such that $\left(x_{2} / d, y_{2} / d\right)$, is on the unit circle [1: $\mathrm{p}$. 17]. These definitions give a distance function [1: pp. 17-18, 3: pp. 225-228]. For $l_{p}$ geometries, the second definition gives

$$
\left|x_{2} / d\right|^{p}+\left|y_{2} / d\right|^{p}=1
$$

so that

$$
d=\left(\left|x_{2}\right|^{p}+\left|y_{2}\right|^{p}\right)^{1 / p}
$$

There are many applications of $l_{p}$ geometries. The shape

$$
|x / a|^{p}+|y / b|^{p}=1
$$

is called a Lamé curve after some work by Gabriel Lamé. Ruane and Swartzlander [5] considered apertures for light with shape (2) with $p>2$, which give a larger area than $p=2$ for their constraints. Piet Hein designed a large traffic island for Stockholm, Sweden using (2) with $p=2.5$ and $a=1.2 b$, saying that it gives a smooth traffic flow. He called the curves (2) with $p>2$ superellipses. The shape (2) has been extensively used for furniture design and elsewhere [6: pp. 240-254]. The Melior typeface's "O" has $p=2.7581$, perhaps for aesthetic reasons.

In the next section, we define normality in Minkowski geometries. Since the normal line to the normal line of a line is usually not the original line, in Section 3 we determine the behavior of the lines obtained by successively finding normal lines of normal lines. The limiting behavior is in Theorems 3.4 and 3.5. In Section 4, we create a circle, called a Radon curve, using portions of two $l_{p}$ geometries' unit circles, for which the normal to the normal of any line is the original line, which is called reflexivity of normality.

\section{Definition of Normality}

There are two equivalent, intuitive ways to define normality in Minkowski geometries with smooth unit circles. One is that line $L_{2}$ is normal to the given line $L_{1}$ with $L_{2}$ meeting $L_{1}$ at point $Q$ if for every point $P$ on line $L_{2}$, the distance from $P$ to $Q$ is the minimum of all distances from $P$ to any point on $L_{1}$ [1: p. 78, 3: p. 228].

For easier expression, we give the other definition in terms of unit vectors. It says that a unit vector is normal to a second unit vector if the first vector contains the origin and a point where the slope of the unit circle is the same as the slope of the second vector [1: p. 125, 7: p. 145]. An application of this definition is illustrated in Figure 2, where $p=6$. We use the second definition, since in practice finding the tangent lines to (1) is easier than minimizing a distance.

In $l_{p}$ geometries, the axes $x=0$ and $y=0$ are mutually normal lines, as are $y=x$ and $y=-x$. However, in general, the normal line to the normal line of a line is not the original line.

In any Minkowski geometry, the unit circle is strictly convex if and only if normality is unique [1: p. 257, 3: p. 232]. If the unit circle contains a line segment $S$, then normality is not unique for any line parallel to that segment. Take such a line $L$ through the origin. Any line through the origin and intersecting $S$ is normal to $L$, since the distance from the origin to the segment is one for all the normal lines. Hence, we do not consider $l_{1}$ or $l_{\infty}$ geometries.

\section{Repeatedly Finding Normal Lines}

The purpose of this section is to explore the behavior of the lines found by repeatedly finding normal lines in $l_{p}$ geometries. The origin $O$ is placed at the point on the initial line where the normal is found.

Lemma 3.1 Consider $l_{p}$ geometry with $p>1$. For $m>0$, the slope of the normal line to $y=m x$ is

$$
-(1 / m)^{1 /(p-1)} \text {. }
$$

For $m<0$, the slope of the normal line to $y=m x$ is

$$
(-1 / m)^{1 /(p-1)} \text {. }
$$

Proof. For $m>0$, we find the point of tangency to the unit circle, where the tangent is parallel to $y=m x$. See Figure 3. In the second quadrant, the derivative of

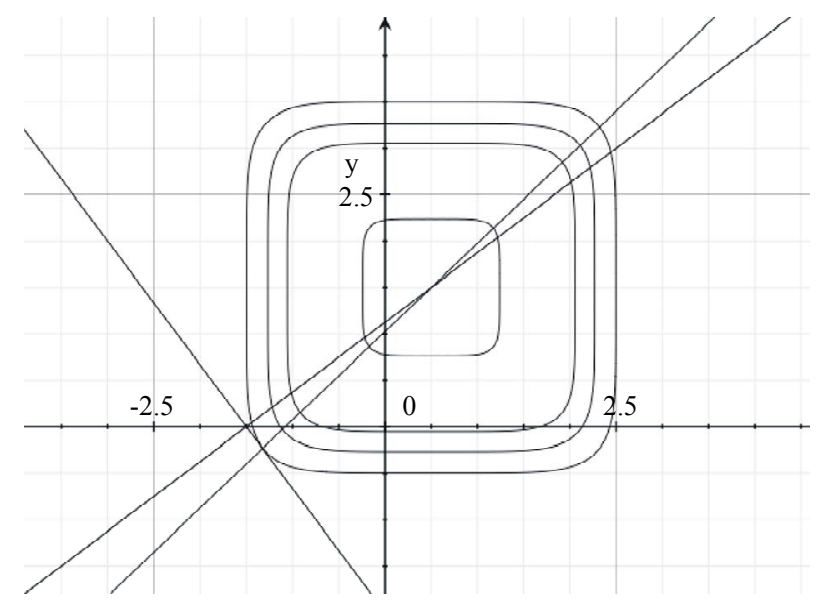

Figure 2. In $I_{6}$ geometry, the line orthogonal to $y=-(4 / 3) x$ -2 through the point $(0.5,1.5)$ is $y=0.95 x+1.025$, but the Euclidean $\left(l_{2}\right)$ normal line is $y=(3 / 4) x+9 / 8$. 


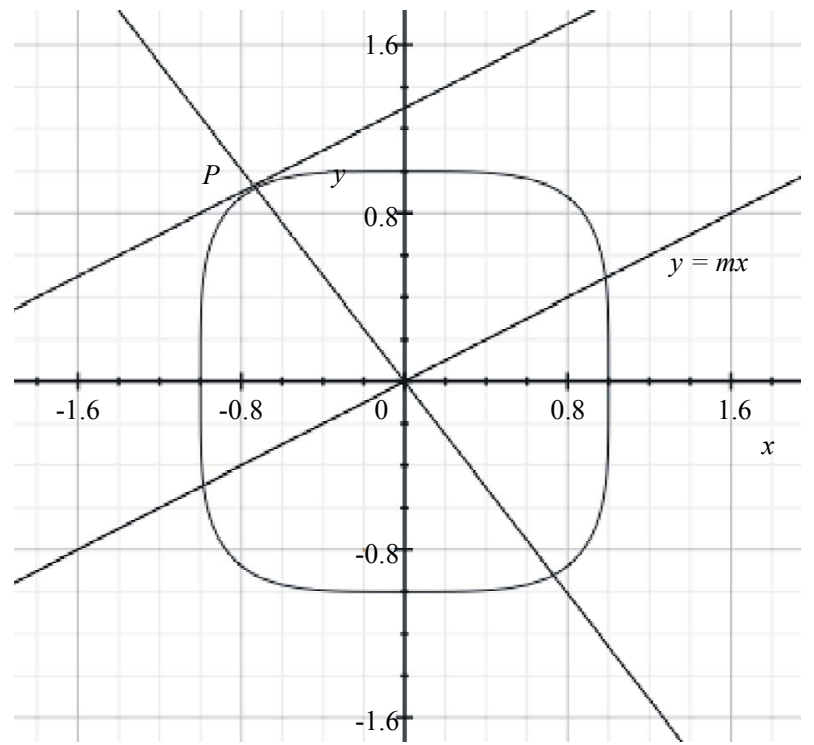

Figure 3. $O P$ is normal to $y=m x$.

$(-x)^{p}+y^{p}=1$ gives $\mathrm{d} y / \mathrm{d} x=(-x / y)^{p-1}$. Setting this equal to $m$ gives $y / x=-(1 / m)^{1 /(p-1)}$, which is the slope of the normal line to $y=m x$. Formula (4) is derived similarly.

Lemma 3.2 Consider $l_{p}$ geometry with $p>1$. Designate by $m_{n}$ the slope of the $n^{\text {th }}$ line found by iteratively finding normal lines at the origin, starting with the line $y=m_{0} x$ with $m_{0}>0$. For even $n$,

$$
m_{n+2}=\left(m_{n}\right)^{1 /(p-1)^{2}}
$$

and for odd $n$,

$$
m_{n+2}=-\left(-m_{n}\right)^{1 /(p-1)^{2}} .
$$

For even $n$,

$$
m_{n}=\left(m_{0}\right)^{1 /(p-1)^{n}},
$$

and for odd $n$,

$$
m_{n}=-\left(1 / m_{0}\right)^{1 /(p-1)^{n}} .
$$

These formulas can be appropriately altered for $m_{0}<0$.

Proof. To obtain (5), for even $n$, using (3),

$$
m_{n+1}=-\left(1 / m_{n}\right)^{1 /(p-1)}
$$

and using (4) and (9),

$$
\begin{aligned}
m_{n+2} & =\left(-1 / m_{n+1}\right)^{1 /(p-1)} \\
& =\left[-1 /\left\{-\left(1 / m_{n}\right)^{1 /(p-1)}\right\}\right]^{1 /(p-1)} \\
& =\left(m_{n}\right)^{1 /(p-1)^{2}} .
\end{aligned}
$$

Equation (5) supplies $m_{2}=\left(m_{0}\right)^{1 /(p-1)^{2}}$ and also the main induction step to give (7). Similarly, obtain (6) and (8). $\square$

Lemma 3.3 Consider $l_{p}$ geometry with $p>1$. If

$$
m_{0,1}=1 / m_{0,2},
$$

then

$$
m_{n, 1}=1 / m_{n, 2},
$$

where the second subscript indicates the identity of the line.

Proof. Take $m_{0,1}$ and $m_{0,2}$ to be positive. The proof for negative initial slopes is similar. For even $n$, using (7) for line 1, (10), and then (7) for line 2 give

$$
m_{n, 1}=\left(m_{0,1}\right)^{1 /(p-1)^{n}}=\left(1 / m_{0,2}\right)^{1 /(p-1)^{n}}=1 / m_{n, 2} .
$$

The proof of (11) for odd $n$ uses (8) and (10) in a similar manner. $\square$

Because of the symmetries of the $l_{p}$ unit circle about the axes, only $m_{0}>0$ need be considered. The condition $m_{0,1}=1 / m_{0,2}$ between the slopes of two initial lines means that the lines have the same angle with the respective axes. Lemma 3.3 shows the symmetries about $y= \pm x$ in the behavior of the iterated normal lines, so only initial slopes between 0 and 1 need to be considered.

Theorem 3.4 Consider $l_{p}$ geometry with $p>2$. For the initial line $y=m_{0} x$ with $0<m_{0}<1$, the subsequence of $\left\{m_{n}\right\}$ for even $n$ has values $0<m_{n}<1$ and monotonically approaches 1 , and the subsequence for odd $n$ has values $m_{n}<-1$ and monotonically approaches -1 . For the initial line $y=m_{0} x$ with $m_{0}>1$, the subsequence of $\left\{m_{n}\right\}$ for even $n$ has values $m_{n}>1$ and monotonically approaches 1 , and the subsequence for odd $n$ has values $m_{n}>-1$ and monotonically approaches -1 .

Proof. Take $0<m_{0}<1$. Using Lemma 3.2, for even $n$,

$$
m_{n+2}=\left(m_{n}\right)^{1 /(p-1)^{2}}>m_{n}
$$

and

$$
\underset{n \rightarrow \infty}{\operatorname{Limit}} m_{n}=\operatorname{Limit}_{n \rightarrow \infty}\left(m_{0}\right)^{1 /(p-1)^{n}}=1 .
$$

For odd $n$,

$$
m_{n+2}=-\left(-m_{n}\right)^{1 /(p-1)^{2}}>m_{n}
$$

and

$$
\underset{n \rightarrow \infty}{\operatorname{Limit}} m_{n}=\operatorname{Limit}_{n \rightarrow \infty}\left\{-\left(1 / m_{0}\right)^{1 /(p-1)^{n}}\right\}=-1 .
$$

Lemma 3.3 says that initial lines $y=m_{0} x$ with $0<m_{0}<1$ give the behavior of the iterated normal lines for $m_{0}>1$. 
As an example of Theorem 3.4, Table 1 contains the slopes of the first eight iterated normal lines for $p=2.5$ with $m_{0}=1 / 5$ and $m_{0}=5$. Lemma 3.3 says that the entries in the table's two columns are inverses, since the values of the $m_{0} \mathrm{~s}$ are inverses. The normal lines monotonically approach the lines $y= \pm x$, as shown by the arrows in their graphs in Figures $\mathbf{4}$ and $\mathbf{5}$.

Theorem 3.5 Consider $l_{p}$ geometry with $1<p<2$. For the initial line $y=m_{0} x$ with $0<m_{0}<1$, the subsequence of $\left\{m_{n}\right\}$ for even $n$ has values $0<m_{n}<1$ and monotonically approaches 0 , and the subsequence for odd $n$ has values $m_{n}<-1$ and monotonically approaches $-\infty$. For the initial line $y=m_{0} x$ with $m_{0}>1$, the subsequence of $\left\{m_{n}\right\}$ for even $n$ has values $m_{n}>1$ and monotonically approaches $\infty$, and the subsequence for odd $n$ has values $m_{n}>-1$ and monotonically approaches 0 .

Table 1. The slopes of the first eight iterated normal lines for $p=2.5$.

\begin{tabular}{lcc}
\hline & $m_{0}=1 / 5$ & $m_{0}=5$ \\
\hline $\boldsymbol{m}_{\mathbf{1}}$ & -2.9240 & -0.3120 \\
$\boldsymbol{m}_{2}$ & 0.4890 & 2.0448 \\
$\boldsymbol{m}_{3}$ & -1.6111 & -0.6207 \\
$\boldsymbol{m}_{4}$ & 0.7277 & 1.3743 \\
$\boldsymbol{m}_{5}$ & -1.2361 & -0.8090 \\
$\boldsymbol{m}_{6}$ & 0.8682 & 1.1518 \\
$\boldsymbol{m}_{7}$ & -1.1000 & -0.9101 \\
$\boldsymbol{m}_{\mathbf{8}}$ & 0.9391 & 1.0648 \\
\hline
\end{tabular}

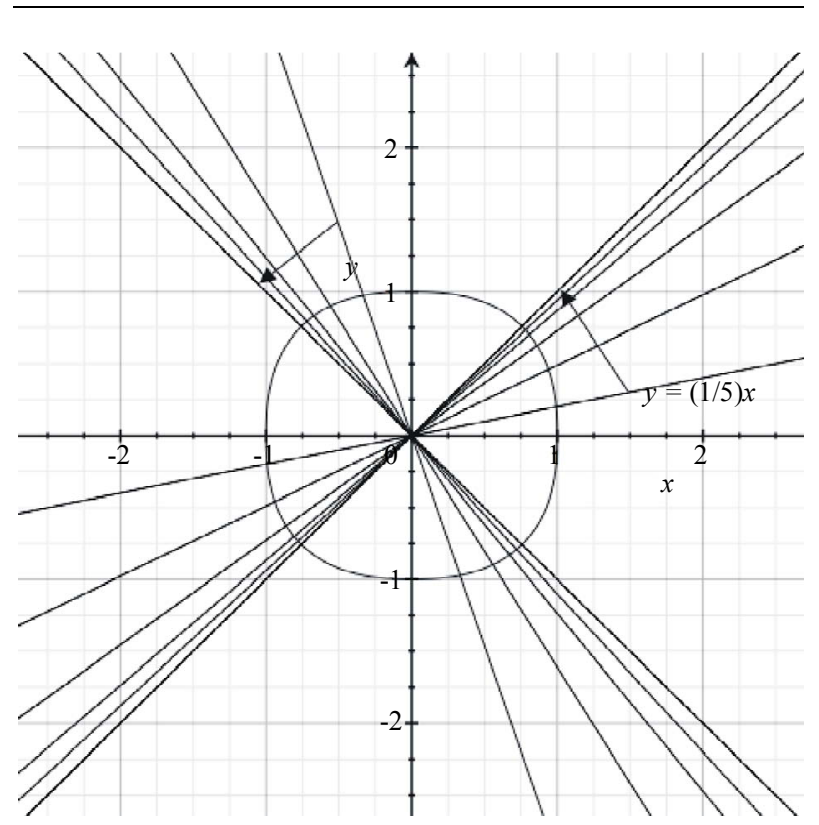

Figure 4. The lines $y=m_{n} x$ for the values in the first column of Table 1 for $p=2.5$ and $m_{0}=1 / 5$.

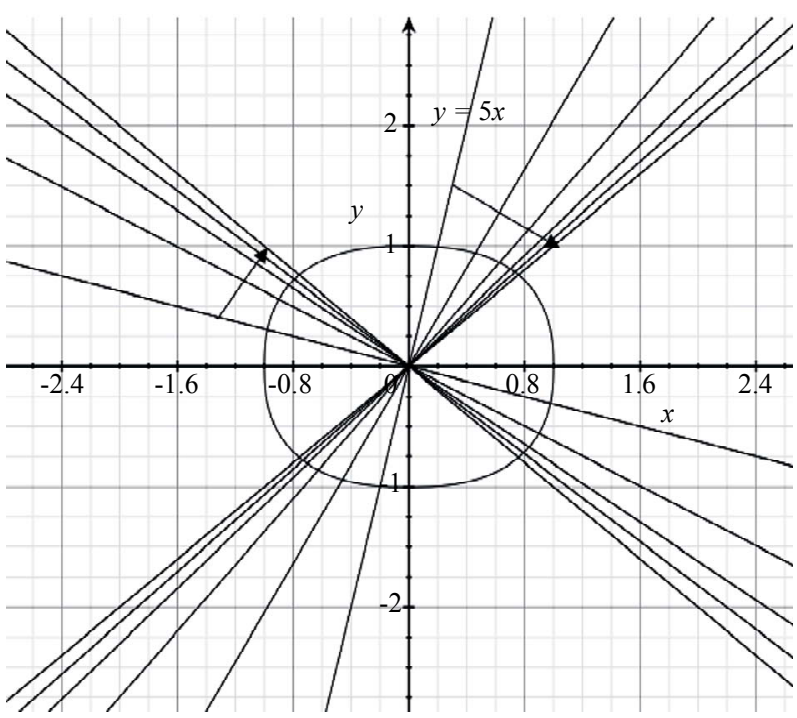

Figure 5. The lines $y=m_{n} x$ for the values in the second column of Table 1 for $p=2.5$ and $m_{0}=5$.

Proof. The proof is the same as the proof of Theorem 3.4 with the small necessary changes being made. $\square$

As an example of Theorem 3.5, Table 2 contains the slopes of the first eight iterated normal lines for $p=5 / 3$ with $m_{0}=4 / 5$ and $m_{0}=5 / 4$. Lemma 3.3 says that the entries in the two columns are inverses, since the values of the $m_{0} \mathrm{~s}$ are inverses. The normal lines monotonically approach the axes, as shown by the arrows in their graphs in Figure 6. The clockwise arrows are for $m_{0}=4 / 5$, and the counterclockwise arrows are for $m_{0}=5 / 4$.

The $l_{p}$ geometries have unit circles that are symmetric about the lines $x=0, y=0, y=x$, and $y=-x$. Theorems 3.4 and 3.5 show that these directions are like attractors or else isolated pairs when iteratively taking normal lines. Taking $x=0$ or $y=0$ as the initial line gives a cycle of normal lines of period 2 between $x=0$ and $y=0$. Taking $y=x$ or $y=-x$ as the initial line gives a cycle of normal lines of period 2 between $y=x$ and $y=-x$.

\section{A Geometry with Reflexive Normality}

Although our focus is on $l_{p}$ geometries with $p>1$, portions of the unit circles (1) for different values of $p$ can be joined to obtain interesting geometries. Theorem 4.1 shows how to make normality reflexive for all lines, that is, the normal to the normal of a line is the initial line. Reflexivity is sometimes called symmetry.

Theorem 4.1 Given the portion of the $l_{p}$ unit circle that is in the first and third quadrants, the only way to complete a unit circle in the second and fourth quadrants for a Minkowski geometry with reflexive normality is with the portions of the $l_{q}$ unit circle in the second and fourth quadrants for $1 / p+1 / q=1$. 
Table 2. The slopes of the first eight iterated normal lines for $p=5 / 3$.

\begin{tabular}{lcc}
\hline & $m_{0}=4 / 5$ & $m_{0}=5 / 4$ \\
\hline $\boldsymbol{m}_{\mathbf{1}}$ & -1.3975 & -0.7155 \\
$\boldsymbol{m}_{\mathbf{2}}$ & 0.6053 & 1.6521 \\
$\boldsymbol{m}_{\mathbf{3}}$ & -2.1236 & -0.4709 \\
$\boldsymbol{m}_{\mathbf{4}}$ & 0.3231 & 3.0946 \\
$\boldsymbol{m}_{5}$ & -5.4439 & -0.1837 \\
$\boldsymbol{m}_{6}$ & 0.0787 & 12.702 \\
$\boldsymbol{m}_{7}$ & -45.269 & -0.0221 \\
$\boldsymbol{m}_{8}$ & 0.0033 & 304.58 \\
\hline
\end{tabular}

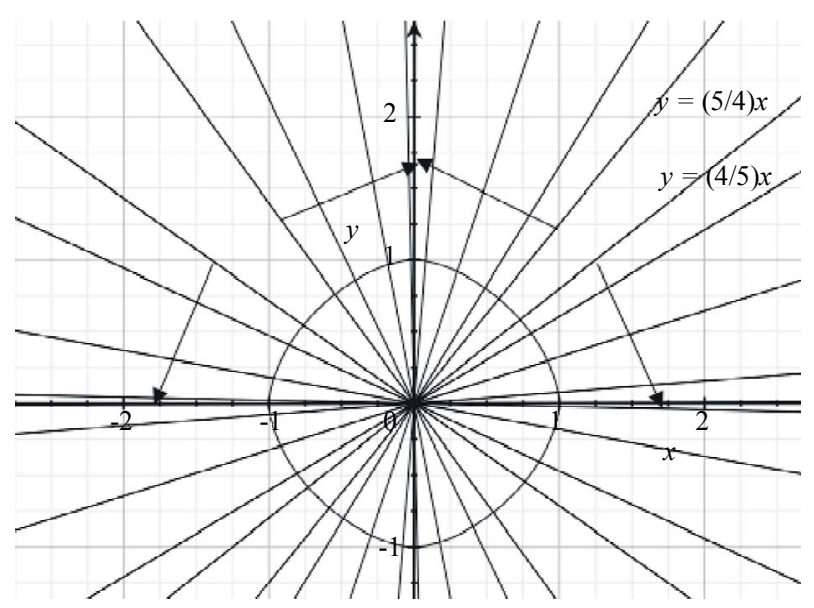

Figure 6. The lines $y=m_{n} x$ for the values in Table 2 for $p=5 / 3$ with $m_{0}=4 / 5$ and $m_{0}=5 / 4$.

Proof. Since Minkowski unit circles are symmetric about their centers, we can reference only the first and second quadrants. Take the center to be the origin, and construct all normal lines at the origin. In the first quadrant, the unit circle is $x^{p}+y^{p}=1$. In the second quadrant, the unit circle is $y=g(x)$. The original line $L_{1}$ is $y=t x, t>0$, which intersects $x^{p}+y^{p}=1$ at the point $P_{1}\left(x_{1}, y_{1}\right)$. The construction is illustrated in Figure 7 for $p=4$. For reflexivity, demand that the slope of line $L_{1}$ equals the slope of the tangent line $L_{3}$ at the point $P_{2}\left(x_{2}, y_{2}\right)$ with $y_{2}=g\left(x_{2}\right)$, and demand that the slope of the line $L_{2}$ tangent to $x^{p}+y^{p}=1$ at $P_{1}\left(x_{1}, y_{1}\right)$ equals the slope $y_{2} / x_{2}$ of the line $L_{4}$, which is to be orthogonal to line $L_{1}$. The goal is to find the function $g(x)$. The slope of $L_{2}$ is found by taking the derivative of $x^{p}+y^{p}=1$ to obtain

$$
p x^{p-1}+p y^{p-1} \mathrm{~d} y / \mathrm{d} x=0 .
$$

Then,

$$
\mathrm{d} y / \mathrm{d} x=-(x / y)^{p-1}=-\{x /(t x)\}^{p-1}=-1 / t^{p-1}=-t^{1-p} .
$$

Equating the slopes of the lines $L_{1}$ and $L_{3}$ gives

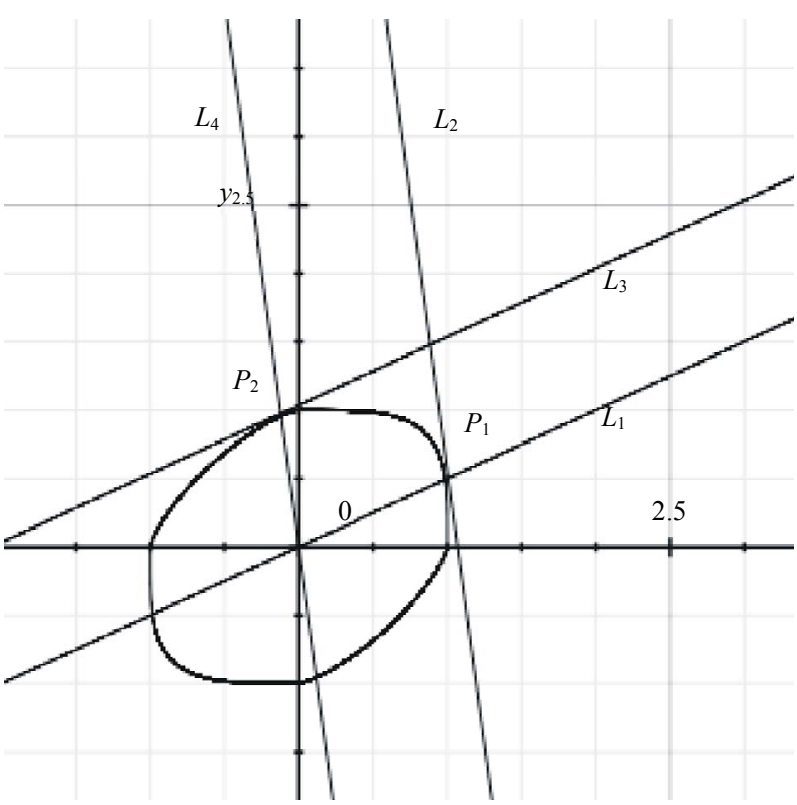

Figure 7. The unit circle in this Minkowski geometry is $|x|^{4}+|y|^{4}=1$ in quadrants 1 and 3 and $|x|^{4 / 3}+|y|^{4 / 3}=1$ in quadrants 2 and 4 . Lines $L_{1}$ and $L_{3}$ are parallel, as are lines $L_{2}$ and $L_{4}$. In this geometry, normality is reflexive, that is, $L_{4}$ is normal to $L_{1}$ and $L_{1}$ is normal to $L_{4}$ for any choice of $L_{1}$.

$$
t=\mathrm{d} y / \mathrm{d} x\left(x_{2}\right)
$$

with $y=g(x)$. Equating the slopes of lines $L_{2}$ and $L_{4}$ gives

$$
-t^{1-p}=y_{2} / x_{2}
$$

Solving (13) for $t$ gives

$$
t=\left(-y_{2} / x_{2}\right)^{1 /(1-p)} .
$$

Equating the expressions for $t$ in (12) and (14) and dropping the subscript 2 give the differential equation

$$
\mathrm{d} y / \mathrm{d} x=(-y / x)^{1 /(1-p)} \text { or } y^{1 /(p-1)} \mathrm{d} y / \mathrm{d} x=(-x)^{1 /(p-1)},
$$

whose unique solution is

$$
y^{p /(p-1)}=-(-x)^{p /(p-1)}+C .
$$

Since $y(0)=g(0)=1, C=1$. Designating $p /(p-1)$ by $q$ gives $1 / p+1 / q=1$ and

$$
|x|^{q}+|y|^{q}=1
$$

for $y=g(x)$ in the second and fourth quadrants. $\square$

The unit circles $|x|^{4}+|y|^{4}=1$ and $|x|^{4 / 3}+|y|^{4 / 3}=1$ are dual, since $1 /(4)+1 /(4 / 3)=1$. Dual unit circles and dual spaces are central to Minkowski geometry $[1,3,7]$.

Schäffer's theorem says that dual unit circles have the same circumferences, when the circumferences are measured with their own distance functions [1: pp. 111-118, 
7: p. 153, 8,9]. Because of the symmetry of the unit circle in Theorem 4.1, it has the same circumference as the dual $l_{p}$ and $l_{q}$ unit circles whose arcs compose it.

Radon curves are equivalently defined as either unit circles for which normality is reflexive for all lines or unit circles that have arcs of dual circles in alternating quadrants as in Theorem 4.1's example [1: p. 128, 3: pp. 233-234, 7: pp. 143-145, 10].

\section{REFERENCES}

[1] A. C. Thompson, "Minkowski Geometry," Cambridge University Press, Cambridge, 1996. http://dx.doi.org/10.1017/CBO9781107325845

[2] E. F. Krause, "Taxicab Geometry," Dover Publications, New York, 1986.

[3] R. V. Benson, "Euclidean Geometry and Convexity," McGraw-Hill, New York, 1966.

[4] B. V. Dekster, "An Angle in Minkowski Space," Journal of Geometry, Vol. 80, No. 1, 2004, pp. 31-47.
[5] G. J. Ruane and G. A. Swartzlander Jr., "Optical Vortex Coronagraphy with an Elliptical Aperture," Applied Optics, Vol. 52, No. 2, 2013, pp. 171-176. http://dx.doi.org/10.1364/AO.52.000171

[6] M. Gardner, "Mathematical Carnival," Alfred A. Knopf, New York, 1975.

[7] J. C. Álvarez Paiva and A. Thompson, "On the Perimeter and Area of the Unit Disc," The American Mathematical Monthly, Vol. 112, No. 2, 2005, pp. 141-154. http://dx.doi.org/10.2307/30037412

[8] J. J. Schäffer, "The Self-Circumferences of Polar Convex Disks," Archiv de Mathematik, Vol. 24, 1973, pp. 87-90. http://dx.doi.org/10.1007/BF01228179

[9] J. B. Keller and R. Vakil, " $\pi_{p}$, the Value of $\pi$ in $l_{p}$," The American Mathematical Monthly, Vol. 116, No. 10, 2009, pp. 931-935. http://dx.doi.org/10.4169/000298909X477069

[10] J. Radon, "Über eine Besondere Art Ebener Konvexer Kurven," Berichte der Sächsische Akademie der Wissenschaften zu Leipzig, Vol. 68, 1916, pp. 131-134. 